\title{
Penetration of molten silicon into a bed of fines
}

\author{
Graham P. Benham ${ }^{a}$, Kjetil Hildal ${ }^{b}$, Colin P. Please ${ }^{a}$, Robert A. Van Gorder ${ }^{a *}$ \\ ${ }^{a}$ Mathematical Institute, University of Oxford, Andrew Wiles Building, Radcliffe Observatory Quarter, Woodstock Road, Oxford OX2 6GG United Kingdom \\ ${ }^{b}$ Elkem Technology, Fiskaaveien 100, 4621 Kristiansand, Norway \\ *Robert.VanGorder@maths.ox.ac.uk
}

\begin{abstract}
During the casting process of silicon the mould must be buffered from the high temperatures of the molten silicon to prevent the mould melting and this is typically done by the operators laying down a layer of crushed silicon particles (fines) prior to pouring of the molten silicon. It is useful for operators to know how deep they should make the layer the fines so as to adequately separate the molten silicon from the mould. In this paper, we consider a model for the penetration of molten silicon into the pre-laid layer of silicon fines, which provides a predictive tool for estimating the necessary depth of fines in order to prevent the molten silicon touching the mould. The mathematical model developed here considers the flow of molten silicon as a Darcy flow and solidification due to heat flow as a one-phase Stefan problem. We are able to find a numerical solutions to this model, and from this we are able to extract data regarding the penetration depth of the molten silicon into the fines before solidification occurs. Our model and numerical solution can been seen as a first step toward understanding this important part of the casting process for silicon.
\end{abstract}

Keywords: solidification of silicon, phase transitions, fines, casting process, Stefan problem

\section{Introduction}

It is known from the literature on solidification of pure silicon and silicon alloys that the cooling rate and mould structure will influence the microstructure of the obtained solid; see [1] and [2]. To prevent the mould melting during the casting of silicon, operators typically lay down a layer of crushed silicon fines prior to pouring of the molten silicon so as to buffer the mould from the high temperatures of the molten silicon. It is useful for operators to know how deep they should lay the fines so as to adequately separate the molten silicon from the mould. Therefore, in the present paper we construct a mathematical model to predict the depth of penetration of molten silicon into the fines layer which is laid down on the mould surface before pouring of the hot liquid melt. This could provide a useful tool for operators to know how deep they should lay their fines so as to separate the molten silicon from the mould, particularly since it is known that interactions with the fines can modify the microstructure obtained from that which might be expected from simply considering the rate of cooling [3]. There is also interest in expanding the understanding of silicon fines behaviour such as applications in the solar energy sector $[4,5]$.

The basic approach taken here is to assume that the fines act as a solid porous material and that the molten silicon then travels through the pore structure until it cools and solidifies. The aim is to determine the movement of the molten silicon and in particular the distance that the molten silicon travels into the porous structure. In practical situations the molten silicon is only just above its melting temperature so melting of the porous structure can be neglected but the initially cold porous structure causes the molten silicon to solidify as it flows and thereby slow the flow down. Furthermore it is anticipated that the flow into the pores can be assumed to have a sharp interface between those pores that are filled with silicon and those that have yet to be filled. This will enable a saturated model of the flow to be used. Similar problems have been studied in relation to the percolation of water through snow $[6,7,8,9]$. The main difference is that in those situations the fluid flow typically includes nonsaturated effects and, more importantly, the snow is only just below freezing and is insufficiently cold to completely freeze the water and close the pores. In contrast, here the fines are initially cold enough to allow the latent heat from the molten silicon to be completely removed and hence solidify the entire system.

The remainder of the paper is as follows. In Section 2, we shall outline the formulation and geometry of the fines problem. Then, in Section 3, we cast the relevant physical problem in terms of a Stefan problem. In Section 4, we give a type of similarity solution for the Stefan problem. From this solution, we are able to extract the salient features of the problem, in order to determine the penetration depth of molten silicon into the fines before solidification occurs. We discuss the results in Section 5, and mention possible directions for future work including the extension to more complicated scenarios that may arise in practical applications.

\section{Formulation}

To allow analytical progress and simple insight to be gained the porous material made by the fines will be assumed to be approximated by a series of isolated cuts in a solid silicon material and furthermore that problem will be taken to be two di- 


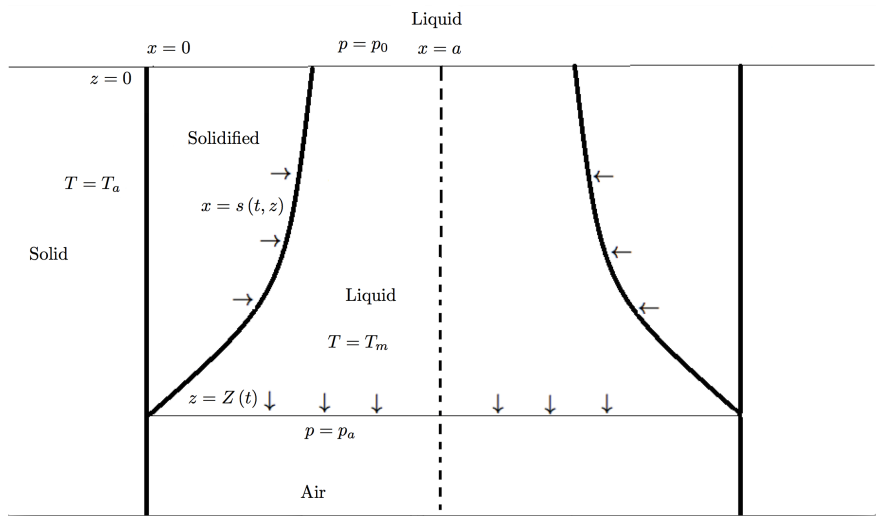

Figure 1: Schematic of the model. Molten silicon penetrates a thin channel between two solid silicon particles. The liquid moves down the channel with its lower interface at $z=Z(t)$. Meanwhile the solidification front $x=s(t, z)$ moves into the channel as heat flows into the surrounding particles.

mensional so the holes can be considered as channels. These channels will be taken to be long and thin, corresponding to a layer of fines that is many particles thick.

Consider the idealised geometry and the notation set out in Figure 1. The molten silicon enters a narrow channel with the walls representing the fines made of solid silicon. Because the liquid is typically only just above the melting point assume that the liquid is at the solidification temperature, $T_{m}$, and that the solid is initially at air temperature, $T_{a}$. The molten silicon will flow into the channel due to the pressure created by overlying pool of static molten silicon which we shall assume gives a known pressure $p_{0}$ at the inlet of the channel. There is a free surface between the liquid fluid and the underlying air that moves down the channel. The details of the free surface will be complicated by the wetting of the solid silicon by the liquid silicon but here we shall simply assume that this surface is horizontal and at atmospheric $p_{a}$ (we assume the air can easily escape from the region).

Take $z$ as the vertical coordinate (pointing down the channel) and $x$ as the horizontal coordinate. The channel entrance is at $z=0$ and there is a the free surface between the molten silicon and the air in the channel which is assumed to be a horizontal surface given by $z=Z(t)$. By symmetry we only need to consider one half of the channel so take $x=0$ to be the solid wall and $x=a$ to be the channel centreline. Furthermore, we neglect the finite horizontal size of the particles and assume that the solid extends to minus infinity in $x$. The liquid will solidify, resulting in an interface between solid and liquid which we denote by $x=s(t, z)$.

To study the problem assume first that the movement of the liquid down the channel is known so that $Z(t)$ is given (and more importantly that the inverse $Z^{-1}(z)$ is known) and so only heat flow need be examined. We will subsequently derive a model for $Z(t)$ by considering the fluid flow. Hence we start by examining the heat dynamics of the silicon and particularly the movement of the interface $x=s(t, z)$.

\section{The Heat Flow Problem}

For simplicity it has been assumed that the molten silicon is very close to the melting temperature, $T_{m}$ and the silicon is very pure. In such a case it is not necessary to consider any complicated phase diagram behaviour so there will be a sharp interface as the material solidifies and only the latent heat of solidification and the diffusion of heat in the solid need to be accounted for. We use Fourier's law for the heat flux and conservation of energy to justify the use of the heat diffusion equation (see [10] for details). This is a free boundary problem (in particular it is a one-phase Stefan problem) where the phase boundary, which separates the solid silicon from the molten silicon, must be found as part of the problem. At the phase boundary the temperature of the silicon will be at the melting temperature and the interface moves according to the Stefan condition which can be derived from an argument of conservation of energy (see [11] for details). The problem is symmetric about the line $x=a$, so we expect a phase boundary to emerge from each wall of the narrow channel. Hence we need only consider one half of the channel, and therefore only one phase boundary. Without loss of generality, we shall consider the region $x \in(-\infty, a]$ and let the position of the phase boundary be denoted $x=s(t, z)$.

The heat flow in the solid is governed by the two-dimensional heat equation

$$
\rho c_{p} \frac{\partial T}{\partial t}=k \nabla^{2} T
$$

where $\rho, c_{p}$, and $k$ are the density, specific heat capacity and thermal conductivity of the material respectively, which are all taken to be constant. In the narrow channel, however, the diffusion distance is much shorter than the channel length so that the heat flow in the solid is approximately governed by the onedimensional heat equation

$$
\rho c_{p} T_{t}=k T_{x x} .
$$

One consequence of this one-dimensional heat flow approximation is that at any particular position down the channel, $z$, for times before the interface arrives, $t<Z^{-1}(z)$, the temperature will remain at its initial state and there is no solidification so that $s(t, z)=0$. Therefore the heat flow at any position $z$ only needs to considered $t \geq Z^{-1}(z)$.

At the phase boundary the temperature must equal the melting temperature, so

$$
T(s(t, z), z, t)=T_{m} .
$$

We have taken the density to be the same in both the liquid and the solid as the density of silicon only varies by about $2 \%$ at this transition. A list of the values of the various constants can be found in Table 1 .

To change phase, the latent heat, $L$, of the silicon must be removed. The 'Stefan condition' (see [11]) dictates that the latent heat required to move the interface must be removed by the difference between the heat fluxes at either side of the solidification front. Considering that the temperature is constant (at melting temperature) in the liquid region, then we get

$$
\rho L \frac{\partial s}{\partial t}=k \frac{\partial T}{\partial x}
$$




\begin{tabular}{ccc}
\hline Constant & Typical Value & Units \\
\hline$T_{\text {pot }}$ & 323 & $\mathrm{~K}$ \\
$T_{m}$ & 1683 & $\mathrm{~K}$ \\
$T_{0}$ & 1700 & $\mathrm{~K}$ \\
$T_{a}$ & 293 & $\mathrm{~K}$ \\
$d$ & $0.03-0.1$ & $\mathrm{~m}$ \\
$\rho$ & 2533 & $\mathrm{~kg} / \mathrm{m}^{3}$ \\
$L$ & 1798060 & $\mathrm{~J} / \mathrm{kg}$ \\
$k_{s}$ & 43 & $\mathrm{~W} / \mathrm{mK}$ \\
$k_{l}$ & 23.5 & $\mathrm{~W} / \mathrm{mK}$ \\
$c_{s}$ & 970 & $\mathrm{~J} / \mathrm{kgK}$ \\
$c_{l}$ & 970 & $\mathrm{~J} / \mathrm{kgK}$ \\
$h$ & 0.02 & $\mathrm{~m}$ \\
$a$ & 0.002 & $\mathrm{~m}$ \\
$p_{a}$ & 101325 & $\mathrm{~kg} / \mathrm{ms}$ \\
$g$ & 9.8 & $\mathrm{~m} / \mathrm{s}^{2}$ \\
$\mu$ & 0.02 & $\mathrm{~kg} / \mathrm{ms}^{2}$ \\
\hline
\end{tabular}

Table 1: List of Dimensional Parameter values provided by Elkem.

at $x=s(z, t)$.

To solve this problem consider the following scalings for each variable:

$$
t=\frac{\rho L a^{2}}{k_{l}\left(T_{m}-T_{a}\right)} \hat{t}, \quad x=a \hat{x}, \quad T=T_{m}+\left(T_{m}-T_{a}\right) \hat{T} .
$$

so that in non-dimensional form, the problem in the liquid region becomes

$$
\hat{T}=0,
$$

while in the solid and solidified regions, it becomes

$$
\begin{aligned}
& S t \frac{\partial \hat{T}}{\partial \hat{t}}=\frac{\partial^{2} \hat{T}}{\partial \hat{x}^{2}}, \\
& \hat{T}=-1 \quad \text { as } \quad \hat{x} \rightarrow-\infty, \\
& \hat{T}=0 \quad \text { on } \quad \hat{x}=\hat{s}(\hat{t}, \hat{z}) .
\end{aligned}
$$

Here, $S t=\frac{c_{s}\left(T_{m}-T a\right)}{L}$ is the "Stefan number", which measures the ratio of sensible heat to latent heat. The conditions at the interface $\hat{x}=\hat{s}(\hat{t}, \hat{z})$, commonly referred to as the "Stefan condition", become

$$
T=0, \quad \frac{\partial \hat{s}}{\partial \hat{t}}=\frac{\partial \hat{T}}{\partial \hat{x}} .
$$

Finally, since the fines are presumed to be at air temperature initially we have the condition

$$
\hat{T}=-1 \quad \text { at } \hat{t}=\hat{Z}^{-1}(\hat{z}) .
$$

Note the problem above governs the movement of the boundary $\hat{x}=\hat{s}(\hat{t}, \hat{z})$ from time when the fluid interface arrives at position $z$, i.e. $t \geq Z^{-1}(z)$ up until the channel solidifies all the way across and this will first occur at top of the channel $\hat{z}=0$ at some time $t^{*}$ when $\hat{s}\left(t^{*}, 0\right)=1$. After this time no more liquid can enter the channel and no fluid flow occurs. There is then a heat problem to solve but this is not of immediate interest so we do not consider this here.

\section{Solution for the Stefan Problem}

The heat flow problem is a classical one-phase Stefan problem on a semi-infinite region. This can be solved analytically, as shown in [10] by noting that there is a similarity solution. The solution is self-similar if we scale all lengths by the square root of the time. Noting that the problem starts at $t=Z^{-1}(z)$ this implies we take

$$
\eta=\frac{\hat{x}}{\sqrt{\hat{t}-\hat{Z}^{-1}(\hat{z})}}, \quad \lambda=\frac{\hat{s}}{\sqrt{\hat{t}-\hat{Z}^{-1}(\hat{z})}},
$$

where $\eta$ is the new independent variable and the position of the solidification front is given by $\eta=\lambda$ where $\lambda$ is a constant to be found. We now assume the solution is of the form $T=f(\eta)$ and get the boundary value problem

$$
f^{\prime \prime}(\eta)+\frac{S t}{2} \eta f^{\prime}(\eta)=0, \quad f(-\infty)=-1, \quad \text { and } \quad f(\lambda)=0 .
$$

The constant $\lambda$ is determined by the Stefan condition

$$
f^{\prime}(\lambda)=\frac{\lambda}{2}
$$

We can obtain the exact solution to (13), and using this we recover the thermal profile

$$
\hat{T}(\hat{x}, \hat{t})=\frac{1+\operatorname{erf}\left(\frac{\hat{x}}{2} \sqrt{\frac{S t}{\hat{t}-\hat{Z}^{-1}(\hat{z})}}\right)}{1+\operatorname{erf}\left(\frac{\lambda}{2} \sqrt{S t}\right)}-1 .
$$

From this expression, we find that $\lambda$ is determined by the transcendental equation

$$
\sqrt{\frac{S t}{\pi}} e^{-\frac{S t t^{2}}{4}}=\frac{\lambda}{2}\left(1+\operatorname{erf}\left(\frac{\lambda}{2} \sqrt{S t}\right)\right) .
$$

We give a plot of the relation between the parameter $\lambda$ and the Stefan number in Figure 2. For the solidification of silicon, we find that $S t=0.74$ resulting in a value of $\lambda=0.68$. Note that (16) is a non-dimensional relationship which gives $\lambda$ as a nonmonotonic function of St. Worster [12] gives a similar relationship which is monotonic, due to the fact that it is in dimensional form. Having determined the behaviour of the thermal problem by assuming the function $Z^{-1}(z)$ was known and shown that

$$
\text { for } \hat{t} \leq \hat{Z}^{-1}(\hat{z}) \quad \text { we have } \hat{s}(\hat{t}, \hat{z})=0 \text {, }
$$

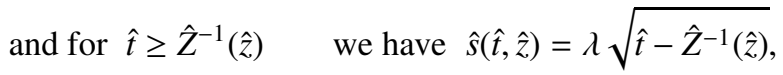

we now return to the problem of determining the fluid flow.

We note that the liquid will move down the channel driven by the pressure difference between the pool of molten silicon above and the air below. We assume the fluid flow is slow with constant viscosity and in a long thin channel with velocity in the horizontal and vertical directions, $u$ and $w$, respectively. We start by considering conservation of mass and note that the vertical velocity has very little $x$ dependence, so we shall assume that $w=w(t, z)$. Conservation of mass gives us

$$
u_{x}+w_{z}=0,
$$




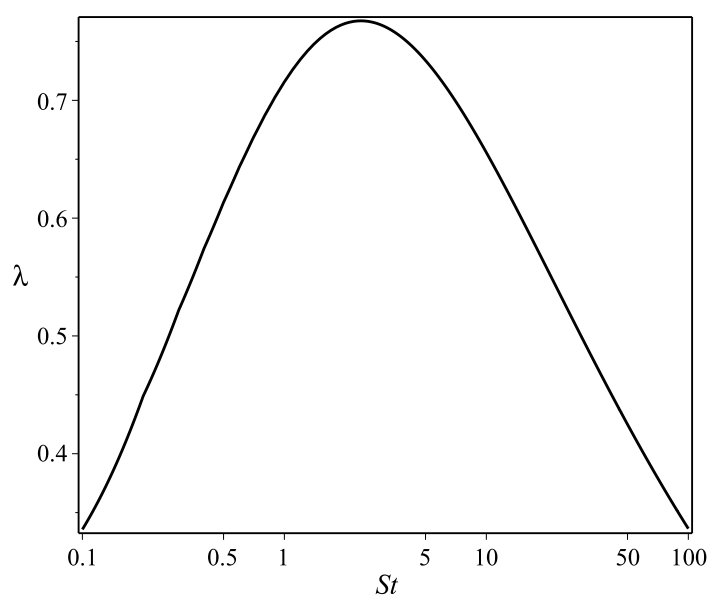

Figure 2: Plot of the relation between the parameter $\lambda$ and the Stefan number, $S t$, obtained from (16). For both of the limits $S t \rightarrow 0$ and $S t \rightarrow \infty$, the value of $\lambda$ approaches zero. For the solidification of silicon, we have $S t=0.74$ resulting in a value of $\lambda=0.68$.

which we integrate over the width of the fluid channel to give us

$$
[u]_{s(t, z)}^{a}+(a-s(t, z)) w_{z}=0 .
$$

By symmetry, we must have $u_{x=a}=0$ and in addition we require a kinematic condition on the solidification front which takes the form

$$
\frac{D}{D t}(s(t, z)-x)=\frac{\partial s}{\partial t} \quad \text { on } \quad x=s(t, z) .
$$

and is equivalent to

$$
\left.u\right|_{x=s(t, z)}=w s_{z} .
$$

Thus, (20) and (22) combine to give the approximate conservation of mass equation

$$
((a-s(t, z)) w(t, z))_{z}=0 .
$$

Note that a simple integration gives

$$
w(t, z)=\frac{Q(t)}{a-s(t, z)},
$$

where $Q(t)$ is the flux down the channel.

We next consider the momentum balance and assume that the slow viscous flow in the channel is governed by Darcy's law

$$
w(t, z)=\frac{-(a-s(t, z))^{2}}{12 \mu} p_{z}
$$

where $p$ is the pressure and $\mu$ is the viscosity. Note in writing this equation down the effects of gravity have been neglected and this is because the pool of molten silicon is generally much deeper that thickness of the layer of fines and hence dominates the gravitational effects.

We now want to solve equations (25) and (24). We assume that the pool of liquid silicon above has depth $H$ and is static so the hydrostatic pressure is gives $p(0, t)=p_{0}=p_{a}+\rho g H$. On the moving interface we assume that are no capillary forces so that

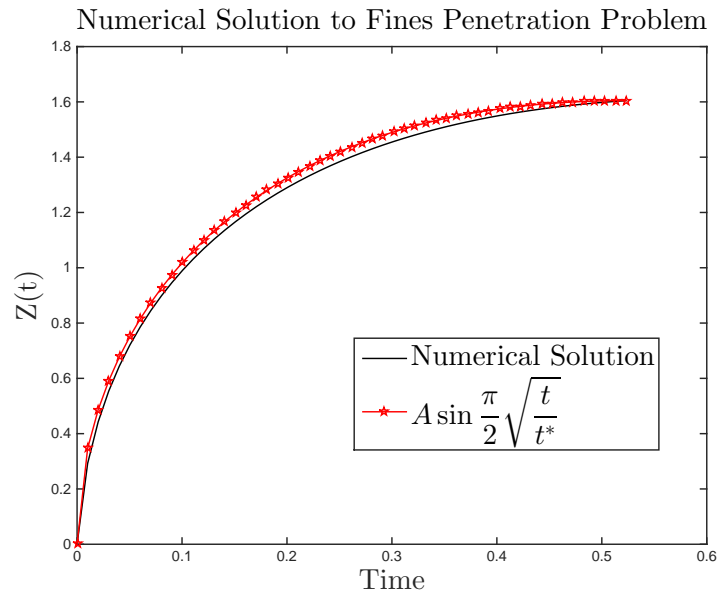

Figure 3: Numerical solution to the non-dimensional ordinary differential equation (30) with initial condition $\hat{Z}(0)=0, \beta=21$ and $S t=0.74$. Note that the solution breaks down at $\hat{t}^{*}=0.52$ because the integral became singular, since the gap at $z=0$ has closed. This is indicated by how $\hat{Z}^{\prime}\left(\hat{t}^{*}\right)=0$. We have also plotted an approximate analytical solution which matches the numerical solution, $Z(t) \approx A \sin \frac{\pi \sqrt{\hat{t}}}{2}$, where $A \approx 1.6$ is the non-dimensional penetration depth. This results in a dimensional penetration depth of $3.2 \mathrm{~cm}$.

$p(Z(t), t)=p_{a}$ and finally that there is a kinematic condition at the moving interface that the velocity of the fluid is the velocity of the interface. This gives

$$
p(t, 0)=p_{0}, \quad p(t, Z(t))=p_{a}, \quad \text { and } \quad \frac{d Z}{d t}=w(t, Z(t)) .
$$

A non-dimensionalised version of the problem can readily be found using the scaling of (5), and we have the scalings

$$
p=p_{a}+\left(p_{0}-p_{a}\right) \hat{p}=p_{a}+\rho g H \hat{p}, \quad Z=H \hat{Z} .
$$

We now substitute $w$ from (24) into (25), impose the condition (26) and exploit the functional form of $s$ found in (12) to give the single equation for $Z(t)$

$$
\frac{d \hat{Z}}{d \hat{t}}=\beta\left(\int_{0}^{\hat{Z}(\hat{t})} \frac{1}{\left(1-\lambda \sqrt{\hat{t}-\hat{Z}^{-1}(\hat{z})}\right)^{2}} d \hat{z}\right)^{-1},
$$

with $\hat{Z}(0)=0$. In deriving this equation we have introduced the non-dimensional constant

$$
\beta=\frac{\rho L a^{4}\left(p_{0}-p_{a}\right)}{12 \mu H^{2} k_{l}\left(T_{m}-T_{a}\right)}
$$

which is the single physical parameter (along with $S t$ ) that governs the movement of the fluid into the fines. It is the ratio of the time taken to solidify a region of thickness $a$ to the time taken to flow down a channel of length $H$.

First note that (28) can be rewritten as

$$
\frac{d \hat{Z}}{d \hat{t}}=\beta\left(\int_{0}^{\hat{t}} \frac{\hat{Z}^{\prime}(u)}{(1-\lambda \sqrt{\hat{t}-u})^{3}} d u\right)^{-1},
$$




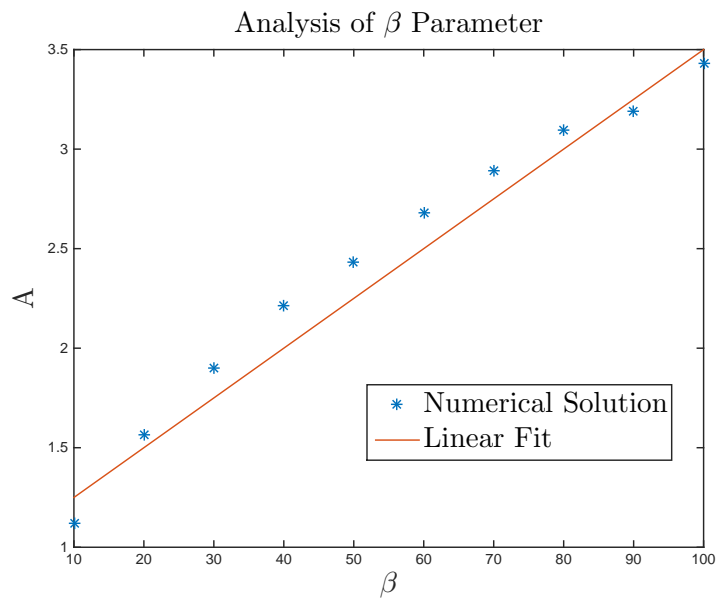

Figure 4: We can see that the ultimate penetration depth appears to increase as we increase the parameter $\beta=\frac{\rho L a^{4}\left(p_{0}-p_{a}\right)}{12 \mu H^{2} k_{l}\left(T_{m}-T_{a}\right)}$. We find that typical values of $\beta$ vary between 10 and 100 . The linear relationship $A=1+0.025 \beta$ appears to fit the data fairly well.

which can be solved numerically. The solution breaks down after a certain time $t=t^{*}$, as by this time the gap at $z=0$ has closed. We plot the numerical solution for $\beta=21$ and $S t=0.74$ in Figure 3. We find that typical values of $\beta$ range between 10 and 100. In Figure 4, we plot the total penetration depth for various values of $\beta$.

Note that at early times, we can expand (30) and show that the behaviour of $Z^{\prime}(t)$ is like $t^{-1 / 2}$ as $t \rightarrow 0$. This is also reflected in the numerical solution (see Figure 3 ) where we see an initially large gradient. From this initial scaling, along with the fact that the solution must eventually reach a maximal value. There is no closed form analytical solution to the equation but by guessing a suitably simple form and fitting it to the numerical solution we find

$$
Z(t)=A \sin \left(\frac{\pi}{2} \sqrt{\frac{t}{t^{*}}}\right)
$$

where $A \approx 1.6$ accurately reproduces the solution for $S t=$ $0.74, \beta=21$, which is seen in Figure 3. This corresponds to a dimensional penetration depth of $3.2 \mathrm{~cm}$. The relationship between $\beta$ and $A$ can be seen in Figure 4. It appears to be linear relationship of the form $A \approx 1+0.025 \beta$.

\section{Conclusions}

We have developed a simple initial model for the penetration of molten silicon into a pre-laid layer of fines which acts as a buffer between the melt and the mould. We were able to find a numerical solution to this initial model which, upon calibration and further analysis, could potentially provide a predictive tool for estimating the necessary depth of fines in order to separate the molten silicon from the mould. Therefore, our model and numerical solution can been seen as a first step toward understanding this process.

In the future, more complicated three-dimensional simulations could be generated, in addition to experimental work. This would allow predictions that accounted in more detail for the geometry of the fines, the capillary forces and any impurities in the silicon but at the expense of significantly greater computational effort. It would also be possible to use these initial ideas to motivate understanding on how the initial grain structured of the fines influences the grain structure of the completed cast. These are therefore interesting and potentially useful areas of further work.

\section{Acknowledgments}

This publication is based on work supported by the EPSRC Centre for Doctoral Training in Industrially Focused Mathematical Modelling (EP/L015803/1) in collaboration with Elkem. G.P. Benham thanks Elkem for financial support and the opportunity to work on-site during parts of this project.

\section{References}

[1] Schei, A., Tuset, J.K., and Tveit, H. 1998. Production of high silicon alloys. Tapir Trondheim, Norway.

[2] Forwald, K.R. 1997. Properties of some silicon rich alloys. NTNU Trondheim Metallurgisk Institutt 127, 147.

[3] Anglezio, J. C., Servant, C., and Dubrous, F. 1990. Characterization of metallurgical grade silicon. Journal of Materials Research 5(09), 18941899.

[4] Filtvedt, W. O., Javidi, M., Holt, A., Melaaen, M. C., Marstein, E., Tathgar, H., and Ramachandran, P. A. 2010. Development of fluidized bed reactors for silicon production. Solar Energy Materials and Solar Cells 94(12), 1980-1995.

[5] Ceccaroli, B. and Lohne, O. 2003. Solar Grade Silicon Feedstock, in Handbook of Photovoltaic Science and Engineering (eds A. Luque and S. Hegedus), John Wiley \& Sons, Ltd, Chichester, UK. doi: 10.1002/0470014008.ch5

[6] Colbeck, S.C. 1975. A Theory for Water Flow Through a Layered Snowpack. Water Resources Research 11(2), 261-266.

[7] Gray, J.M.N.T. 1996. Water Movement in Wet Snow. Philosophical Transactions of the Royal Society London A 354, 465-500.

[8] Morland, L.W., Kelly, R.J., and Morris, E.M. 1990. A mixture theory for a phase-changing snowpack. Cold Regions Science and Technology $17,271-285$

[9] Tseng, P., Illangasekare, T.H., and Meier, M.F. 1994. Modelling of snow melting and uniform wetting front migration in a layered subfreezing snowpack. Water Resources Research 30, 2363-2376.

[10] Crank, J. 1979. The Mathematics of Diffusion. Oxford University Press

[11] Rubinstein, L.I. 1971. The Stefan Problem. 27 American Mathematical Society.

[12] Worster, M.G. 2000. Solidification of fluids. Perspectives in fluid dynamics $742,393-446$. 